\title{
THE PRODUCTION OF ELEVATED FLIGHT FORCE COMPROMISES MANOEUVRABILITY IN THE FRUIT FLY DROSOPHILA MELANOGASTER
}

\author{
FRITZ-OLAF LEHMANN AND MICHAEL H. DICKINSON* \\ Theodor-Boveri-Institute, Department of Behavioral Physiology and Sociobiology, University of Würzburg, Am \\ Hubland, 97074 Würzburg, Germany \\ *Present address and address for correspondence: University of California at Berkeley, Valley Life Sciences Building, Department of \\ Integrative Biology, Berkeley, CA 94720, USA (flymanmd@ violet.berkeley.edu)
}

Accepted 4 December 2000; published on WWW 1 February 2001

\begin{abstract}
Summary
In this study, we have investigated how enhanced total flight force production compromises steering performance in tethered flying fruit flies, Drosophila melanogaster. The animals were flown in a closed-loop virtual-reality flight arena in which they modulated total flight force production in response to vertically oscillating visual patterns. By simultaneously measuring stroke amplitude and stroke frequency, we recorded the ability of each fly to modulate

$2.7^{\circ}$ and $4.8 \mathrm{~Hz}$ of their mean value, respectively. This variance in wing kinematics decreases with increasing flight force production, and at maximum force production fruit flies are restricted to a unique combination of stroke amplitude, stroke frequency and mean force coefficient. This collapse in the kinematic envelope during peak force production could greatly attenuate the manoeuvrability and stability of animals in free flight.
\end{abstract} its wing kinematics at different levels of aerodynamic force production. At a flight force that exactly compensates body weight, the temporal deviations with which fruit flies vary their stroke amplitude and frequency are approximately

Key words: steering capacity, course control, manoeuvrability, flight, force production, fruit fly, Drosophila melanogaster.

\section{Introduction}

Insects display an impressive variety of flight manoeuvres (Dalton, 1975; Nachtigall, 1974). Flies, in particular, are capable of extraordinary aerial behaviors (Collett and Land, 1975; Land and Collett, 1974; Wagner, 1985; Wagner, 1986; Wehrhahn et al., 1982) aided by an array of unique sensory specializations including neural superposition eyes and gyroscopic halteres (Braitenberg, 1967; Dickinson, 1999; Hengstenberg, 1998; Kirschfeld, 1967; Nalbach, 1994; Pringle, 1948; Zeil, 1983). Using this elaborate sensory input, flies steer and manoeuvre by changing many aspects of their wing kinematics including the angle of attack (Zanker, 1990), the amplitude and frequency of the wing stroke (Götz, 1968; Götz, 1983; Götz et al., 1979; Lehmann and Dickinson, 1998) and the timing and speed of wing rotation (Dickinson et al., 1993; Dickinson et al., 1999; Ennos, 1989; Götz, 1987; Lehmann, 1994; Lehmann, 2000). The limits of these kinematic alterations, and thus the constraints on the aerial manoeuvrability of a fly, depend on several factors including the maximum power output of the flight muscles, mechanical constraints imposed by the thoracic exoskeleton and the ability of the underlying neuromuscular system to control the wing movements.

Within the last few decades, the flight control and manoeuvrability of flies have been studied using a variety of methods in both free (Bülthoff et al., 1980; Collett and Land,
1975; Ennos, 1989; Wagner, 1985; Wagner, 1986; Wehrhahn et al., 1982) and tethered (Hollick, 1940; Lehmann and Dickinson, 1998; Zanker and Götz, 1990) flight conditions. Free-flight measurements are critical because they capture the behavior of an animal in a more natural context. However, under such conditions, it is difficult to measure the alterations in wing kinematics, forces and moments that are necessary to construct a satisfying mechanistic explanation of manoeuvrability. This problem can be partly circumvented using tethered animals flown under closed-loop visual feedback. While the flight performance and steering capacity determined in such experiments may reflect only a small fraction of the insect's total behavioral repertoire in free flight, the technique has proved useful in elucidating the organization of the flight control system in flies (Götz, 1968; Heisenberg and Wolf, 1979; Heisenberg and Wolf, 1993).

In flying insects, both the production and the control of flight forces reside in the complex motion with which the animals move their wings. For this reason, the structures that limit maximum total flight force production in an insect should also limit the animal's capacity to steer and, thus, its aerial manoeuvrability. In this paper, we attempt to quantify the potential trade-off between peak performance and manoeuvrability in Drosophila melanogaster using a closedloop flight simulator. By manipulating visual flow in a vertical 
direction, we induced the animals to vary their total flight force production while simultaneously recording their ability to modulate force in response to horizontal visual stimuli. The results indicate that the modulation of total flight force compromises the steering performance and manoeuvrability of flying insects.

\section{Materials and methods Animals}

The data in this paper were collected from 27 2- to 5-dayold female Canton S wild-type fruit flies Drosophila melanogaster (Meigen). The animals were originally obtained from the Drosophila National Species Resource Center (Bowling Green, Ohio, USA) and reared on commercial Drosophila medium (Carolina Biological) at room temperature $\left(22^{\circ} \mathrm{C}\right)$. The tested animals had a mean body mass of $0.65 \pm 0.06 \mathrm{mg}$ (mean \pm S.D.). Unless stated otherwise, all reported values represent mean values \pm S.D. Some of the force measurements and kinematic data presented here have been used previously in an analysis of flight performance (Lehmann and Dickinson, 1997; Lehmann and Dickinson, 1998).

\section{Virtual-reality flight arena}

We have previously provided a more detailed description of the experimental apparatus (Lehmann and Dickinson, 1997) and give only a brief outline here. The flies were tethered and flown in a virtual-reality flight arena in which the stroke amplitudes of both wings, stroke frequency and total flight force were simultaneously sampled at $8.3 \mathrm{~Hz}$ using an AXOTAPE data-acquisition system (Axon Instruments). Under closed-loop feedback conditions, the fly controls the angular velocity of a $30^{\circ}$ wide vertical dark bar displayed in the arena by changing the stroke amplitude of its two wings. While the fly actively stabilized the position of the dark stripe in the frontal region of its visual field, we oscillated a superimposed pattern of diagonal stripes in the vertical direction. In response to this open-loop pattern motion, fruit flies modulate their total flight force production in order to minimize the induced retinal slip. Throughout the following description, a value of $0^{\circ}$ flight direction indicates that the stripe is positioned directly in front of the fly, whereas positive and negative angles indicate object positions to the right and left of the animal, respectively. The term 'stroke amplitude' defines the average amplitude of the two beating wings. The terms 'maximum performance' and 'minimum performance' describe the extreme $1 \%$ of all values within the flight sequence during which the flies produced maximum and minimum flight force, respectively. We use the term 'hovering conditions' to describe the portion of the flight sequence during which the flies produced a flight force within $\pm 1 \%$ of their body weight.

The angular velocity with which the vertical bar moves within the flight arena depends on two factors: the difference between the left and right stroke amplitudes of the animal and the coupling coefficient of the experimental apparatus. In all experiments presented here, the closed-loop coupling coefficient was $61^{\circ} \mathrm{s}^{-1}$ angular velocity of the moving stripe for each degree difference in stroke amplitude between the two wings. This value corresponds to a coupling coefficient of approximately $209^{\circ} \mathrm{s}^{-1} \mathrm{nN}^{-1} \mathrm{~m}^{-1}$ yaw torque in tethered flying fruit flies, assuming a conversion factor of $0.29 \mathrm{nNm}$ yaw torque per degree difference between the left and right stroke amplitudes (Götz, 1983). In comparison, this closedloop coupling coefficient is roughly twice that used in previous closed-loop studies on Drosophila melanogaster $\left(110^{\circ} \mathrm{s}^{-1} \mathrm{nN}^{-1} \mathrm{~m}^{-1}\right.$ yaw torque; Wolf and Heisenberg, 1990), a value chosen so that the torque spikes measured during tethered flight (Heisenberg and Wolf, 1979) would generate a change of the same magnitude in the angular position of the visual panorama as that produced during free-flight saccades (body saccades). Tethered-flight saccades appear to be approximately 5-10 times longer than free-flight saccades, a difference that is thought to be due to the absence of mechanosensory stimuli from the halteres in restrained animals (Heisenberg and Wolf, 1984; Mayer et al., 1988). Heisenberg and Wolf (Heisenberg and Wolf, 1984) adjusted the tethered flight coupling coefficient so that the angular displacement generated by a single torque spike would produce a rotation of $60^{\circ}$ in the visual field. More recent results from free-flight tracking experiments, however, indicate that the saccades produced by freely flying Drosophila melanogaster are closer to $90^{\circ}$ (M. H. Dickinson, in preparation). In our apparatus, $90^{\circ}$ rotations during a wingbeat 'glitch' (the stroke amplitude equivalent of a torque spike) would be generated by a coupling coefficient of $165^{\circ} \mathrm{s}^{-1} \mathrm{nN}^{-1} \mathrm{~m}^{-1}$ yaw torque, which is very close to the value we have used in the present study. Separate experiments in which we have systematically varied the coefficient by a factor of 2 revealed no substantial changes in the flight responses discussed here (F.-O. Lehmann and M. H. Dickinson, in preparation).

\section{Data analysis}

To characterize steering performance in tethered flies, we determined the temporal deviation in stroke kinematics with which the animals modulated their stroke amplitude and frequency in response to the visual patterns. We derived the temporal deviation for each kinematic variable from time traces by calculating the standard deviation from the mean of eight successive data points within a sliding data window (Fig. 1). At the given sampling rate of $8.3 \mathrm{~Hz}$, the width of this window corresponds to a flight time of approximately $1 \mathrm{~s}$. In contrast, the deviation in flight direction was estimated from the width of stripe position histograms. These position histograms were derived from the spatial distribution of the stripe's angular position during flight and calculated for 13 normalized flight force ranges $(0.5-1.7$, in steps of 0.1$)$. The standard deviation in flight direction per flight force bin is then given as 0.849 of half the width of a Gaussian fit to each histogram at half peak height.

To quantify the performance of steering behavior in fruit flies in more detail, we measured the steering capacity, $C_{\mathrm{S}}$, 




Fig. 1. Steering frequency, steering angle and temporal deviation in the angular position of the black stripe within the virtual-reality flight arena as defined in the present study.

which we defined as the average bilateral difference in stroke amplitude per unit time:

$$
C_{\mathrm{S}}=N^{-1} \int_{n=1}^{N} \mathrm{~d} S_{\mathrm{D}} / \mathrm{d} t,
$$

in which $N$ is the number of data samples, $S_{\mathrm{D}}$ is the absolute difference in stroke amplitude between the left and the right wing and $t$ is time. We also determined the frequency of steering commands (steering frequency), which is the number of directional changes of the vertical bar per second. In freely flying animals, this measure would correspond to the frequency with which the animal reverses its flight course from a flight trajectory to the left to a trajectory to the right and vice versa. We estimated the absolute angle between two successive steering commands (steering angle) as a measure of how far the fly would have turned around its body yaw axis under freeflight conditions (Fig. 1). To compare the means in steering performance for minimum, hovering and maximum flight conditions, we used a standard paired $t$-test with which the differences between the three force production conditions were tested statistically (see Fig. 7).

\section{Results}

While steering towards the vertical bar, the flies responded to the vertical oscillation of a superimposed background pattern by altering their total flight force production. As shown in a previous study, these alterations are tightly correlated with changes in stroke amplitude and frequency (Lehmann and Dickinson, 1998). The variance in the data set implies that different combinations of stroke amplitude and stroke frequency can generate the same amount of mean flight force. The variability in stroke kinematics, however, decreases noticeably as flight forces approach maximal values. Fruit flies produce maximum flight force at a unique combination of stroke amplitude and stroke frequency (see Fig. 7 in Lehmann and Dickinson, 1998). A time history of flight force production, stroke kinematics and their temporal variance is shown in Fig. 2. At minimum force production, fruit flies modulate their stroke kinematics as indicated by the high temporal deviation of stroke amplitude and stroke frequency.
In contrast, the temporal deviation of stroke kinematics approaches zero when the animal produces elevated flight forces in response to the upward-moving background stimulus.

Averaging the data for all 27 flies indicates that the temporal deviation of stroke amplitude decreases significantly with increasing aerodynamic force production with a slope of $-2.21 \pm 0.11^{\circ}$ per unit normalized force (model II linear regression, $y=-2.21 x+5.06, r^{2}=0.96, P<0.0001, N=20$ force ranges, Fig. 3A). For hovering conditions, stroke amplitude is $163 \pm 1.5^{\circ}$ and its deviation is approximately $2.7 \pm 0.87^{\circ}$. The temporal deviation in mean stroke frequency changes with total force generation in a manner quite similar to the alteration in stroke amplitude (Fig. 3B). Stroke frequency deviation decreases linearly with increasing force by $-4.57 \pm 0.44 \mathrm{~Hz}$ per unit normalized force (model II linear regression, $y=-4.57 x+9.38, \quad r^{2}=0.84, \quad P<0.0001, \quad N=20$ force ranges, Fig. 3B). At flight forces equal to body weight, fruit flies flap their wings at a frequency of $210 \pm 3.1 \mathrm{~Hz}$, with a temporal deviation of $4.81 \pm 2.17 \mathrm{~Hz}$.

The decrease in kinematic variance with increasing aerodynamic force production compromises the ability of the animal to control thrust, lift and torque. In Drosophila spp., the production of thrust and lift depends linearly both on the mean wing velocity of the flapping wings and on more subtle changes in stroke kinematics that may be lumped collectively into a mean force coefficient (Ennos, 1989; Lehmann and Dickinson, 1998). Previous experiments indicate that fruit flies may use different combinations of wing velocity and mean force coefficient to generate a given amount of flight force. Mean wing velocity, measured at the center of wing area, shows a temporal deviation of $0.042 \pm 0.017 \mathrm{~m} \mathrm{~s}^{-1}$ for hovering conditions and $0.031 \pm 0.01 \mathrm{~m} \mathrm{~s}^{-1}$ at maximum force production (Fig. 4A). The overall slope of wing velocity deviation indicates that this variable decreases significantly with increasing force by $-0.047 \pm 0.002 \mathrm{~m} \mathrm{~s}^{-1}$ per normalized flight force (model II linear regression, $y=-0.047 x+0.091, r^{2}=0.96$, $P<0.0001, N=24$ force ranges). At a temporal deviation in wing velocity equal to zero, fruit flies would produce their flight force at a unique combination of wing velocity and mean force coefficient.

While total force production depends on the mean wing velocity of both wings, fruit flies vary their yaw torque by altering the difference in wing velocity between the two wings (Götz, 1983). Since stroke frequency is identical for both wings, alterations in the angular velocity of the moving vertical bar, and thus flight direction, depend solely on changes in stroke amplitude. By symmetry, the mean difference in wing velocity between the left and the right wings should remain near zero and should be independent of total force production. However, Fig. 4B shows a small leftward bias at elevated force production while the fly is fixating the vertical bar in the frontal region of its visual field (Fig. 5). This asymmetry results from a small difference in the electronic offsets that we used to balance the stroke-amplitude-dependent voltages coming from the left and the right wingbeat analyzer. The flies obviously compensated for this small difference with a unilateral increase 
Fig. 2. Typical flight sequence of a fruit fly showing the temporal deviation in stroke kinematics and flight direction (red) while the animal varies its total aerodynamic force in response to the visual bias (black). In an attempt to stabilize the vertical motion of the bias, the fly varies its total flight force production by modulating its mean stroke amplitude $(\overline{\mathrm{L}+\mathrm{R}})$ and stroke frequency (blue). To keep the vertical bar in front of its visual field (green), the fly modulates the difference in stroke amplitude between the left and right wings (L-R, blue). TD, temporal deviation. See text for further details.
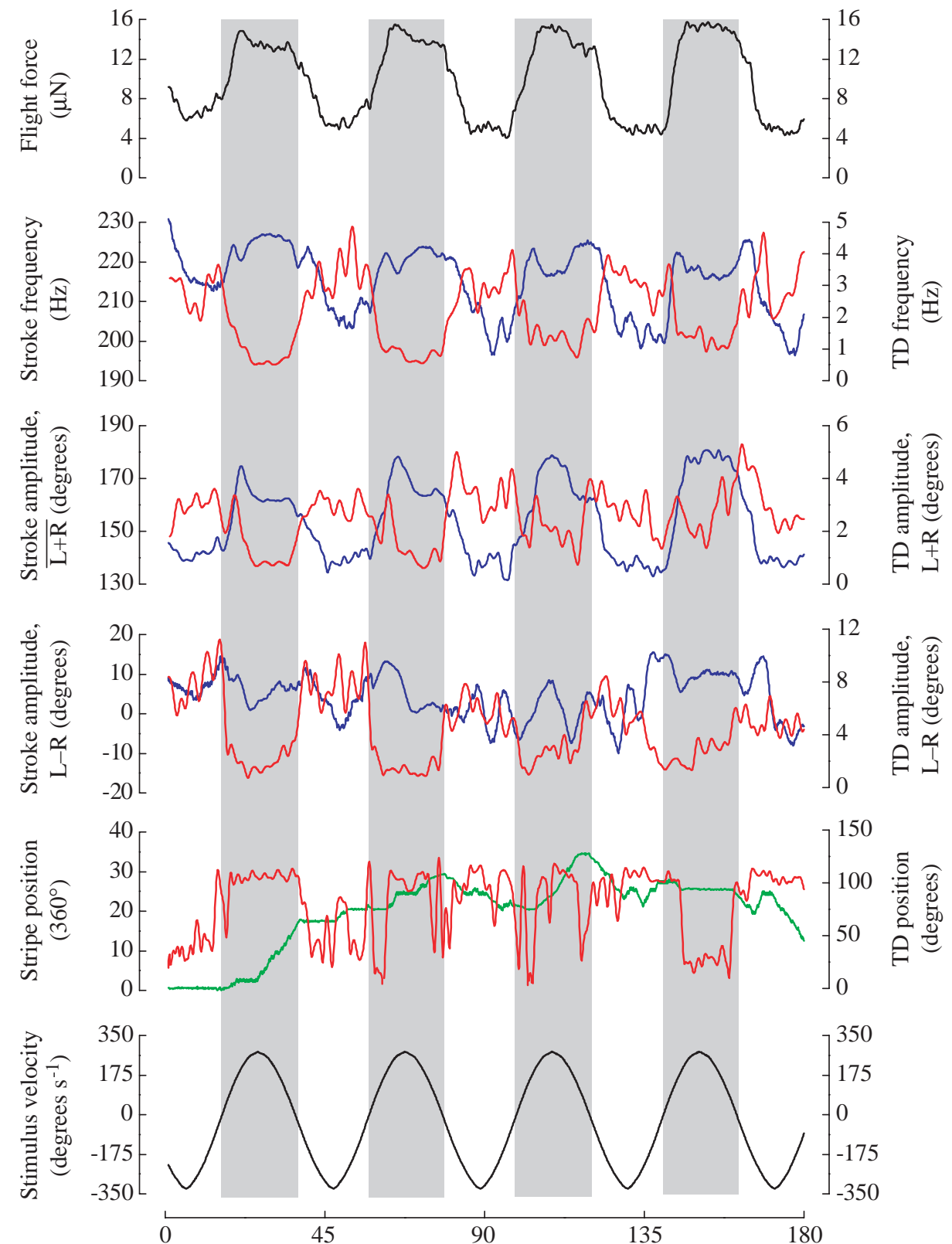

Flight time (s)

Fig. 3. Stroke kinematics (black) and temporal deviation in stroke kinematics (red) plotted as a function of total flight force production. The deviation of both (A) mean stroke amplitude and (B) stroke frequency varies with relative flight force production. The shaded areas indicate the S.D. of the plotted mean values ( $N=27$ flies). TD, temporal deviation.
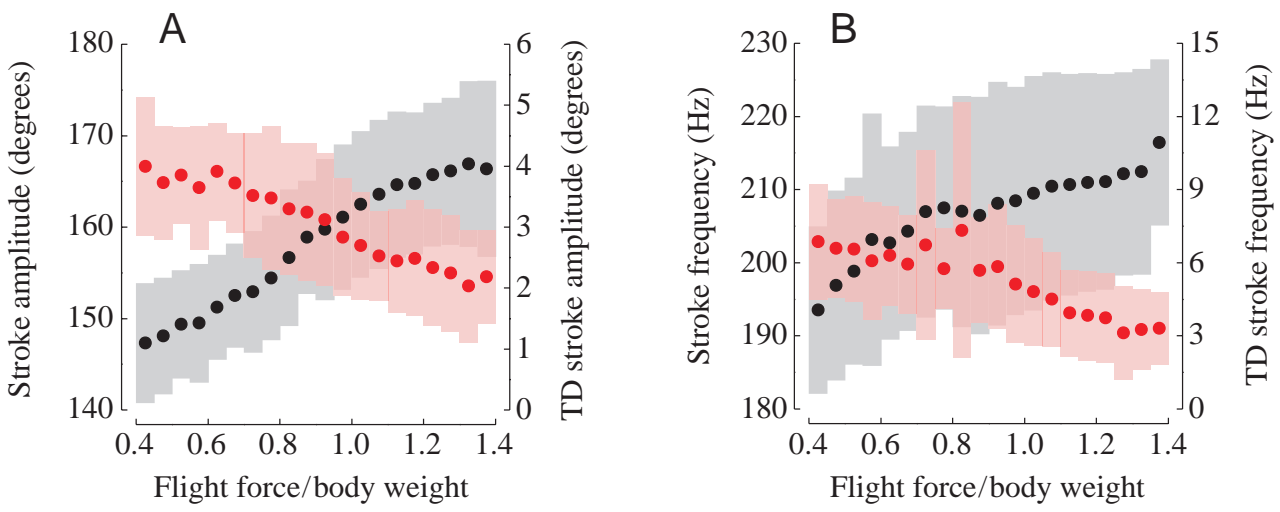
Fig. 4. (A) Variation in mean wing velocity (black) determined at the center of wing area and the temporal deviation of wing velocity (red) with total force production in fruit flies. (B) The difference in mean wing velocity between the left $(\mathrm{L})$ and right $(\mathrm{R})$ wing (black) and its temporal deviation (red) under different aerodynamic loads (B). See text for further details. The shaded areas indicate the S.D. of the plotted mean values ( $N=27$ flies). $\mathrm{TD}$, temporal deviation.

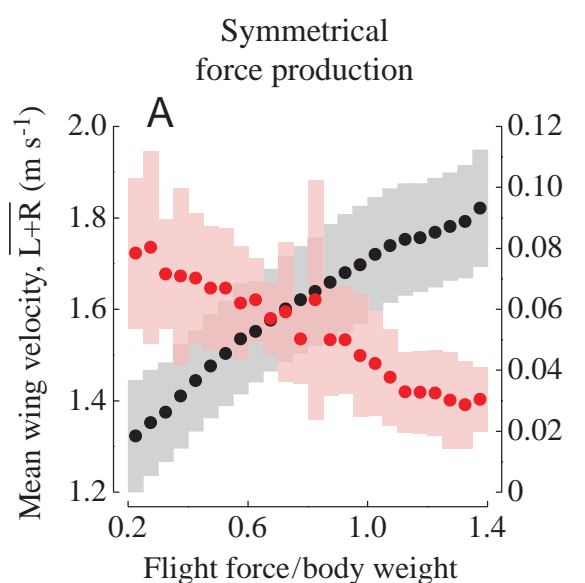

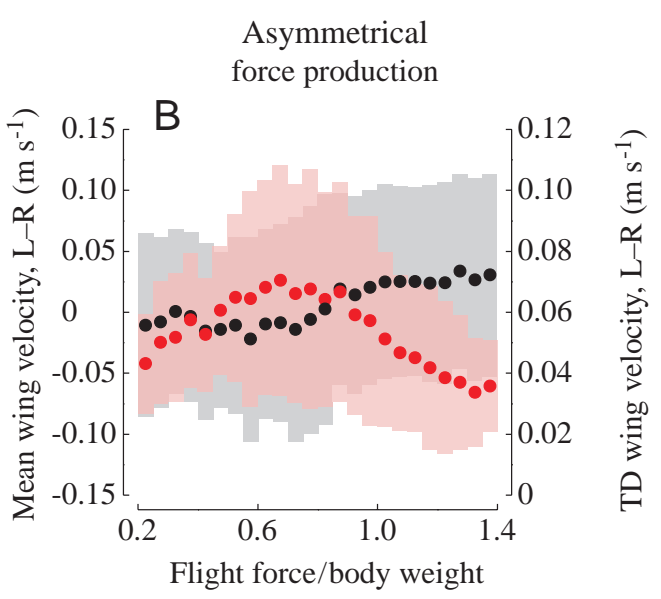

in stroke amplitude that resulted in a slightly higher wing velocity of the left wing. At low forces, the temporal deviation of the wing velocity difference is positively correlated with
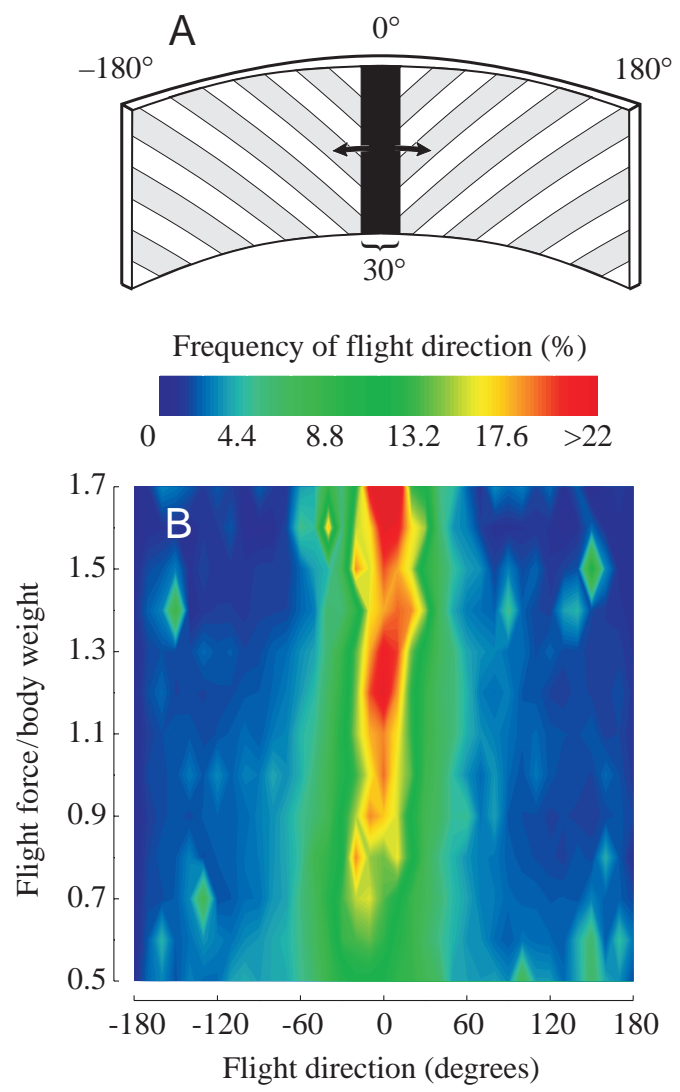

Fig. 5. Changes in the position histograms of the vertical stripe with increasing force production. (A) Stripes of the foreground pattern (black stripe) and the background pattern (chevron-shaped stripes) as displayed in the virtual-reality flight arena. (B) Splined data of stripe position histograms calculated for 13 equally spaced normalized flight force bins ranging from 0.5 to 1.7 . The frequency of flight direction is plotted in pseudo-color code and indicates the probability with which the fly keeps the vertical stripe at a certain angular position within the arena. Values are means for 27 flies. total force production and has a maximum of $0.061 \pm 0.021 \mathrm{~m} \mathrm{~s}^{-1}$ at approximately $70 \%$ normalized force (Fig. 4B). However, if the flies increase their flight force further, the variance of the velocity difference between the two wings is negatively correlated with total force, and variance tends to decrease with increasing force.

Superficially, the decrease in apparent steering performance with increasing force production does not seem greatly to attenuate the fixation behavior towards the vertical bar (Fig. 5). However, a statistical analysis reveals that the standard deviation in flight direction, calculated from the width of the stripe position histograms, decreases significantly with increasing flight forces by $-14.2 \pm 2.2^{\circ}$ per normalized force (model II linear regression, $y=-14.2 x+41.2, r^{2}=0.75, P<0.001$, $N=13$ force ranges) from approximately 37 to $23^{\circ}$ (Fig. 6). At relative flight forces above approximately $70 \%$, this decrease in flight direction deviation is accompanied by a decrease in the animal's steering capacity, which decreases significantly with a slope of $-47.9 \pm 2.1^{\circ} \mathrm{s}^{-1}$ per normalized force (model II linear regression, $y=-47.9 x+81.1, r^{2}=0.99, P<0.0001, N=15$ force ranges, $70-140 \%$ flight force).

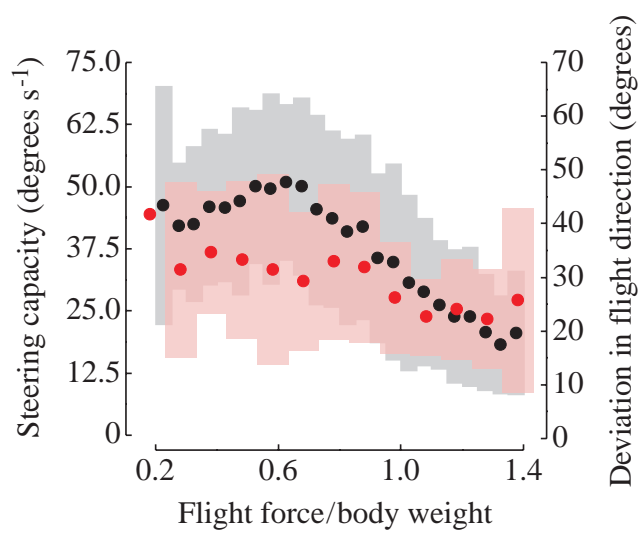

Fig. 6. Variation in steering capacity (black) and standard deviation in flight direction (red) with aerodynamic force production with locomotor activity. The deviation of mean flight direction is given by the width of the position histograms in Fig. 5. The shaded areas indicate the S.D. of the plotted mean values ( $N=27$ flies). 


\section{F.-O. LEHMANN AND M. H. DicKinson}

Fig. 7. Steering behavior and flight direction in tethered fruit flies flying under visual closed-loop feedback conditions. The data were derived for minimum total flight force production (blue), for a flight force equal to the fly's body weight (yellow) and for maximum aerodynamic force (red). (A) Steering frequency derived as the inverse of time between two successive steering commands, (B) mean angular velocity (sign ignored) of the black stripe displayed in the flight arena, (C)


steering angle between two successive flight course reversals, (D) mean flight direction and (E) temporal deviation of the stripe's azimuth position. We excluded from the analysis those flies in which the number of flight course reversals at minimum or maximum force production was less than five. Asterisks indicate significant differences $(* P<0.05, * * P<0.01, * * * P<0.001)$. Values are means + S.D. ( $N=22$ flies). NS, not significant; TD, temporal deviation.

To make a broad comparison between steering behavior in tethered flying fruit flies at different levels of force production, we have summarized steering performance at minimum (blue columns), maximum (red columns) and hovering (yellow columns) flight performance in Fig. 7. These values were derived from the motion of the vertical bar in the arena that the flies controlled under closed-loop feedback conditions. With increasing flight force production, fruit flies do not significantly alter the frequency with which they perform flight course reversals (steering frequency) around their yaw axis (paired $t$-test, $P>0.05$, d.f. $=21$, Fig. 7A). However, the angular velocity with which the bar is moving decreases by a factor of 2 from approximately $422 \pm 223^{\circ} \mathrm{s}^{-1}$ at minimum flight force to roughly $244 \pm 212^{\circ} \mathrm{s}^{-1}$ at maximum force (Fig. 7B). The angle that the bar travels between two successive flight course reversals (steering angle) is shown in Fig. 7C. At minimum force, the vertical bar moves, on average, $91 \pm 67.3^{\circ}$ before the fly reverses its direction. For hovering conditions, steering angle is approximately $41.4 \pm 28.1^{\circ}$. The mean flight direction, calculated by simply averaging all angular positions of the vertical bar within each flight sequence, indicates that the flies keep the bar in the frontal region of their visual field. This measure seems to be rather independent of aerodynamic force production (Fig. 7D). Fig. 7E shows that the mean temporal deviation of the stripe position within a flight sequence, as derived from the sliding data window, decreases with increasing flight force production, a conclusion similar to that we drew from the statistical analysis of the stripe position histograms in Fig. 6. At low force production, the deviation from the mean flight direction is significantly different from those for hovering and maximum force production conditions.

\section{Discussion}

We have investigated the potential trade-off between total force production and steering performance in tethered flying fruit flies Drosophila melanogaster. The data show that in this species the temporal deviation in stroke amplitude and stroke frequency decreases linearly with increasing flight force production (Fig. 3). As a consequence, the variance of both the mean wing velocity and the difference in wing velocity between the left and the right wings decreases with increasing flight force production (Fig. 4). At maximum flight force production, the temporal deviation of both measures approaches zero, indicating that fruit flies are restricted to a unique combination of mean wing velocity and mean force coefficient. This finding suggests that, at maximum locomotor capacity, fruit flies may be substantially compromised in their ability to accelerate or stabilize rotational moments by modulating stroke kinematics (Fig. 7).

\section{Ecological significance of manoeuvrability}

Although many studies on flight control in insects exist in the literature, very few of them have directly determined the ecological advantage of high aerial manoeuvrability. These studies are of great interest because they may directly link the flight behavior of an insect with its reproductive success. High aerial manoeuvrability of an insect may be useful in a large variety of behavioral contexts including predator avoidance, prey catching, mating success and male-male competition. A well-known example of predator avoidance is the evasive flight reactions of noctuid moths when they detect the ultrasound of predating bats (Roeder and Treat, 1961). The success of honeybee (Apis mellifera) drones in catching virgin queens presumably depends in part on their manoeuvrability (Currie, 1987). More direct evidence that the aerial manoeuvrability of an insect may determine its ecological fitness comes from studies on male-male competition in the European beewolf Philanthus triangulum. In this species, behavioral observations suggest a close correlation between flight manoeuvrability and mating success. Male beewolves establish small territories near female nests and defend this territory against other males in air combats, exhibiting fast zigzagging flights (Strohm and Lechner, 2000). Several observations strongly suggest that females most often mate with the owner of the territory and thus with the winner of the combats (Evans and O'Neill, 1988; Simon-Thomas and Poorter, 1972). Aerial performance in terms of load-lifting capacity might also constrain mating 
success in the dance fly Hilara sp. Male dance flies present insect prey to females and initiate copulation while flying tandem with the female and the prey. It seems likely that mating success is limited by the male's capacity to lift its own body mass plus the additional loads (Marden, 1989). A study on muscle maturation in a dragonfly indicates that young dragonflies are poor flyers but gain muscle mechanical power output during adult growth. Maximum power reserves for both flight force production and steering performance are exhibited at maturity. At this age, dragonflies defend a territory, and aerial competition determines mating success (Marden et al., 1998).

\section{Total force production and steering capacity}

The results we present here on the flight behavior of fruit flies imply that any estimation of maximum locomotor capacity in an insect should also consider the potential concomitant loss in manoeuvrability. At elevated force production, this trade-off may compromise the animal's ability to control forces and moments in all six degrees of freedom: the three translational axes, thrust, lift and side-slip, and the three rotational axes, yaw, pitch and roll. To calculate the response of the insect's body to aerodynamic forces produced by the beating wings, or gravitational moments produced by movements of the legs and the abdomen in flight, the moment of inertia and the location of the center of mass of the animal must be estimated (Zanker, 1988). Pitch and roll moments in insects, for example, greatly depend on the center of body mass with respect to the wing base. In Diptera, the distance between these two points is the radius for the first moment of body mass and amounts to approximately $18 \%$ relative body length (Ellington, 1984). During flight, gravity should therefore give the animal a favorable roll and upward pitch moment that passively stabilize the animal's body in an upright position. Besides the large variety of different factors that may contribute to steering behavior and manoeuvrability performance in flying insects, the control of yaw torque seems to be of great importance because it determines the direction in which the animal is moving. Previous studies in flies, moreover, have shown that yaw torque, or its kinematic equivalents, is actively controlled with high temporal accuracy by the underlying neuromuscular system (Heide and Götz, 1996; Lehmann and Götz, 1996; Tu and Dickinson, 1996). Although yaw represents only one degree of freedom of all possible movements a flying animal may achieve, it might be a useful measure for a rough estimate of manoeuvrability in flying insects.

The trade-off between manoeuvrability and elevated force production is especially important for loading experiments in which freely flying insects are tested for their ability to lift added weights (Lehmann, 1999; Marden, 1987). The same argument also holds for estimations of maximum flight performance in insects hovering in heliox (Dudley, 1995). Our data suggest that both experimental procedures might determine the limits of locomotor capacity in terms of maximum aerodynamic force production but may underestimate the functional deficit induced by the challenge to the flight system. Thus, while animals carrying additional loads may be able to get off the ground, a reduced capacity for flight control might render them susceptible to predation or incapable of defending a territory.

\section{Wing kinematics and yaw torque}

Previous studies have shown that, in tethered flying fruit flies, yaw torque is linearly correlated with the difference in stroke amplitude between the left and right wing (Götz, 1983; Lehmann, 1994). However, flies might also control rotational moments by more subtle changes in stroke kinematics including alterations in the angle of attack, the wing trajectory or wing rotation at the end of each half-stroke (Ennos, 1989; Götz, 1987; Zanker, 1990). Measurements in a dynamically scaled robotic insect imply that, for fruit fly kinematics, wing rotation contributes approximately $35 \%$ of the total lift production throughout the stroke (Dickinson et al., 1999). The contribution of rotational circulation, however, depends on the timing with which the wing flip occurs. A flip phase advance by $8 \%$ of the stroke cycle (from rotation that lags stroke reversal to rotation that is synchronous with stroke reversal) increases total lift by $67 \%$. However, during hovering flight in Drosophila spp. and many other insects, the wings beat in an approximately horizontal stroke plane in which yaw torque requires a left-right asymmetry in drag, not in lift. A left-right lift bias would actually create roll moments, depending how the lift production is distributed throughout the stroke. Even assuming a stroke plane inclination of $10-20^{\circ}$ in freely flying fruit flies (Lehmann and Dickinson, 1998), yaw torque would still be affected by changes in drag more than by changes in lift. In the robot, a flip advance by $8 \%$ of the stroke cycle (from rotation that lags stroke reversal to rotation that is synchronous with stroke reversal) increases total drag by approximately $22 \%$, which is significantly smaller than the increase in total lift production. In comparison, tethered flying fruit flies change their wing kinematics during the ventral flip in response to the motion of a single stripe (fixation response) or a stripe grating (optomotor response) by altering the relative timing of wing rotation by $40-70 \mu \mathrm{s}$ (Dickinson et al., 1993) and $69 \mu \mathrm{s}$ (Lehmann, 1994), respectively. At a constant stroke amplitude of $162^{\circ}$ and a stroke frequency of $209 \mathrm{~Hz}$ (hovering conditions), a value of $70 \mu$ s corresponds to an asymmetry of $1.5 \%$ in flip timing, which results in an asymmetry of $13 \%$ in lift and an asymmetry of $4 \%$ in drag between the two wings due to rotational effects (Lehmann and Dickinson, 1998). During the optomotor yaw response, however, fruit flies do not solely advance relative wing rotation but also increase the relative angular velocity of wing rotation by approximately $12 \times 10^{3 \circ} \mathrm{s}^{-1}$ (Lehmann, 1994) and relative stroke amplitude by approximately $9^{\circ}$ (Götz, 1983). Although typically used simultaneously during flight manoeuvres, the animals are able to control their stroke amplitude and the timing of wing rotation independently (Dickinson et al., 1993). This finding suggests that flies might stabilize translational and rotational moments even at elevated force production when stroke 
amplitudes have reached their morphological limits (Lehmann and Dickinson, 1997). The possible contribution of alterations in angular velocity of wing rotation or changes in the wing-tip trajectory to steering performance in flying insects is still under investigation.

\section{Steering frequency and steering angle}

Fig. 7A indicates that steering frequency in Drosophila melanogaster, defined as the inverse of the time between two flight course reversals, is approximately $2 \mathrm{~Hz}$ and is independent of total flight force production. Fourier analysis of closed-loop torque traces measured in the same fly species has revealed a very similar main frequency component in the Fourier spectrum (Wolf and Heisenberg, 1990). In contrast to steering frequency, steering angle, defined as the angle between two successive flight course reversals, decreases by $46 \%$ with increasing flight force, from approximately $91^{\circ}$ at minimum force production to approximately $41^{\circ}$ at a flight force equal to the animal's weight (Fig. 7C). Since steering frequency remains approximately constant, the angular velocity with which the vertical stripe travels between two successive flight course reversals decreases with increasing force in a manner quite similar to steering angle (Fig. 7B). The mean turning angle of $41^{\circ}$ during fixation behavior towards the vertical bar is thought to result from a continuous modulation in yaw torque of approximately $\pm 1.0 \mathrm{nN}$ m peak-to-peak (Heisenberg and Wolf, 1984) that the fly generates by graduated alterations in the bilateral difference in stroke amplitude (Götz, 1983). This aspect of flight control has been investigated in great detail at the level of the underlying neuromuscular system (Heide and Götz, 1996; Lehmann and Götz, 1996; Tu and Dickinson, 1996). However, a yaw torque of $1.0 \mathrm{nN} \mathrm{m}$ is also achieved by a tethered flying fruit fly during uniform yaw torque spikes produced by wingbeat 'glitches' during searching behavior (Heisenberg and Wolf, 1984). A steering angle of $41^{\circ}$ is therefore roughly half the turning angle (41 versus $90^{\circ}$ ) with which a freely flying fly rotates around its yaw axis per body saccade. This might indicate that the closed-loop coupling coefficient of $209^{\circ} \mathrm{s}^{-1} \mathrm{nN}^{-1} \mathrm{~m}^{-1}$ yaw torque we used is too small compared with free flight.

A previous study on closed-loop yaw torque production in Drosophila melanogaster has demonstrated that mean torque amplitude remained constant at approximately $0.5 \mathrm{nN}$ m while the coupling coefficient was altered between 55 and $440^{\circ} \mathrm{s}^{-1} \mathrm{nN}^{-1} \mathrm{~m}^{-1}$ yaw torque (Wolf and Heisenberg, 1990). The same study also showed that, during optomotor balance, the duration of half-wave torque pulses changed only slightly when the coupling coefficient was changed repeatedly between 55 and $165^{\circ} \mathrm{s}^{-1} \mathrm{nN}^{-1} \mathrm{~m}^{-1}$. Moreover, most of this change in duration was transient and largely disappeared within the first $2 \mathrm{~s}$ of altering the feedback conditions. Collectively, the above observations suggest that, in closed-loop flight of Drosophila melanogaster, the torque pulses are broadly independent of the coupling coefficient. As a consequence, tethered fruit flies might achieve a closed-loop steering angle of $90^{\circ}$ (free-flight turning angle) at a coupling coefficient as high as $459^{\circ} \mathrm{s}^{-1} \mathrm{nN}^{-1} \mathrm{~m}^{-1}$ yaw torque, which is significantly larger than the coefficient used in the present analysis.

In this study, we have attempted to characterize the tradeoff between flight force production and manoeuvrability in tethered flying fruit flies. Our analysis suggests that the high locomotor capacity of a flying insect should be regarded both as a power reserve for carrying loads and as a locomotor reserve to ensure steering performance. Since the production of aerodynamic forces and the control of translational forces and rotational moments reside in the same neuromuscular and mechanical structures, the flight-related fitness of an insect may be determined not only by the animal's maximum power reserve but also by how the animal copes with the trade-off between elevated force production and manoeuvrability.

We would like to thank the two unknown referees for their helpful comments on this manuscript. This project was funded by a grant Le905/4 of the German Science Foundation (DFG).

\section{References}

Braitenberg, V. (1967). Patterns of projection in the visual system of the fly. I. Retina-lamina projections. Exp. Brain Res. 3, 271-298.

Bülthoff, H., Poggio, T. and Wehrhahn, C. (1980). 3-D-analysis of the flight trajectories of flies (Drosophila melanogaster). $Z$. Naturforsch. 35c, 811-815.

Collett, T. S. and Land, M. F. (1975). Visual control of flight behavior in the hoverfly, Syritta pipiens L. J. Comp. Physiol. A 99, $1-66$.

Currie, R. W. (1987). The biology and behavior of drones. Bee World 68, 129-143.

Dalton, S. (1975). Borne on the Wind. New York: Reader's Digest Press.

Dickinson, M. (1999). Haltere mediated equilibrium reflexes of the fruit fly, Drosophila melanogaster. Phil. Trans. R. Soc. Lond. 354, 903-916.

Dickinson, M. H., Lehmann, F.-O. and Götz, K. G. (1993). The active control of wing rotation by Drosophila. J. Exp. Biol. 182, 173-189.

Dickinson, M. H., Lehmann, F.-O. and Sane, S. (1999). Wing rotation and the aerodynamic basis of insect flight. Science $\mathbf{2 8 4}$, 1881-2044.

Dudley, R. (1995). Extraordinary flight performance of orchid bees (Apidae: Euglossini) hovering in heliox $\left(80 \% \mathrm{He} / 20 \% \mathrm{O}_{2}\right)$. J. Exp. Biol. 198, 1065-1070.

Ellington, C. P. (1984). The aerodynamics of insect flight. II. Morphological parameters. Phil. Trans. R. Soc. Lond. B 305, $17-40$.

Ennos, A. R. (1989). The kinematics and aerodynamics of the free flight of some Diptera. J. Exp. Biol. 142, 49-85.

Evans, H. E. and O'Neill, K. M. (1988). The Natural History of North American Beewolves. Ithaca, NY: Cornell University Press.

Götz, K. G. (1968). Flight control in Drosophila by visual perception of motion. Kybernetik 4, 199-208.

Götz, K. G. (1983). Bewegungssehen and Flugsteuerung bei der Fliege Drosophila. In BIONA- Report 2 (ed. W. Nachtigall), pp. 21-34. Stuttgart: Fischer.

Götz, K. G. (1987). Course control, metabolism and wing 
interference during ultralong tethered flight in Drosophila melanogaster. J. Exp. Biol. 128, 35-46.

Götz, K. G., Hengstenberg, B. and Biesinger, R. (1979). Optomotor control of wing beat and body posture in Drosophila. Biol. Cybernetics 35, 101-112.

Heide, G. and Götz, K. G. (1996). Optomotor control of course and altitude in Drosophila is achieved by at least three pairs of flight steering muscles. J. Exp. Biol. 199, 1711-1726.

Heisenberg, M. and Wolf, R. (1979). On the fine structure of yaw torque in visual flight orientation of Drosophila melanogaster. J. Comp. Physiol. A 130, 113-130.

Heisenberg, M. and Wolf, R. (1984). Vision in Drosophila. Berlin: Springer-Verlag.

Heisenberg, M. and Wolf, R. (1993). The sensory-motor link in motion-dependent flight control of flies. In Visual Motion and its Role in the Stabilization of Gaze (ed. F. A. Miles and J. Wallman), pp. 265-283. Amsterdam, London, New York, Tokyo: Elsevier.

Hengstenberg, R. (1998). Controlling the fly's gyroscope. Nature 392, 757.

Hollick, F. S. J. (1940). The flight of the dipterous fly Muscina sabulans Fallén. Phil. Trans. R. Soc. Lond. B 230, 357-390.

Kirschfeld, K. (1967). Die Projektion der optischen Umwelt auf das Raster der Rhabdomere im Komplexauge von Musca. Exp. Brain Res. 3, 248-270.

Land, M. F. and Collett, T. S. (1974). Chasing behaviour of houseflies (Fannia canicularis). J. Comp. Physiol. A 89, 331-357.

Lehmann, F.-O. (1994). Aerodynamische, kinematische und elektrophysiologische Aspekte der Flugkrafterzeugung und Flugkraftsteuerung bei der Taufliege Drosophila melanogaster. Thesis, University of Tübingen.

Lehmann, F.-O. (1999). Ambient temperature affects free-flight performance in the fruit fly Drosophila melanogaster. J. Comp. Physiol. B 169, 165-171.

Lehmann, F. O. (2000). Flattern für Flugkräfte. Naturwiss. Rundschau 623, 223-230.

Lehmann, F.-O. and Dickinson, M. H. (1997). The changes in power requirements and muscle efficiency during elevated force production in the fruit fly Drosophila melanogaster. J. Exp. Biol. 200, 1133-1143.

Lehmann, F.-O. and Dickinson, M. H. (1998). The control of wing kinematics and flight forces in fruit flies (Drosophila spp.). J. Exp. Biol. 201, 385-401.

Lehmann, F.-O. and Götz, K. G. (1996). Activation phase ensures kinematic efficacy in flight-steering muscles of Drosophila melanogaster. J. Comp. Physiol. 179, 311-322.

Marden, J. (1987). Maximum lift production during take-off in flying animals. J. Exp. Biol. 130, 235-258.
Marden, J. H. (1989). Effects of load-lifting constraints on the mating system of a dance fly. Ecology 70, 496-502.

Marden, J. H., Fitzhugh, G. H. and Wolf, M. R. (1998). From molecules to mating success: Integrative biology of muscle maturation in a dragonfly. Am. Zool. 38, 528-544.

Mayer, M., Vogtmann, K., Bausenwein, B., Wolf, R. and Heisenberg, M. (1988). Flight control during 'free yaw turns' in Drosophila melanogaster. J. Comp. Physiol. A 163, 389-399.

Nachtigall, W. (1974). Insects in Flight. New York: McGraw-Hill.

Nalbach, G. (1994). Extremely non-orthogonal axes in a sense organ for rotation: behavioral analysis of the dipteran haltere system. Neuroscience 61, 149-163.

Pringle, J. W. S. (1948). The gyroscopic mechanism of the halteres of Diptera. Phil. Trans. R. Soc. Lond. B 233, 347-384.

Roeder, K. D. and Treat, A. E. (1961). The detection and evasion of bats by moths. Am. Sci. 49, 135-148.

Simon-Thomas, R. T. and Poorter, E. P. R. (1972). Notes on the behavior of males of Philanthus triangulum (F.) (Hymenoptera, Sphecidae). Tijdschr. Ent. 115, 141-151.

Strohm, E. and Lechner, K. (2000). Male size does not affect territorial behaviour and life history traits in a sphecid wasp. Anim. Behav. 59, 183-191.

Tu, M. S. and Dickinson, M. H. (1996). The control of wing kinematics by two steering muscles of the blowfly, Calliphora vicina. J. Comp. Physiol. A 178, 813-830.

Wagner, H. (1985). Aspects of the free flight behaviour of houseflies (Musca domestica). In Insect Locomotion (ed. M. Gewecke and G. Wendler), pp. 223-232. Hamburg, Berlin: Paul Parey.

Wagner, H. (1986). Flight performance and visual control of flight of the free-flying housefly (Musca domestica L.). II. Pursuit of targets. Phil. Trans. R. Soc. Lond. B 312, 553-579.

Wehrhahn, C., Poggio, T. and Bülthoff, H. (1982). Tracking and chasing in house flies (Musca). An analysis of 3-D flight trajectories. Biol. Cybernetics 45, 123-130.

Wolf, R. and Heisenberg, M. (1990). Visual control of straight flight in Drosophila melanogaster. J. Comp. Physiol. A 167, 269-283.

Zanker, J. M. (1988). How does lateral abdomen deflection contribute to flight control of Drosophila melanogaster. J. Comp. Physiol. A 162, 581-588.

Zanker, J. M. (1990). The wing beat of Drosophila melanogaster. III. Control. Phil. Trans. R. Soc. Lond. B 327, 45-64.

Zanker, J. M. and Götz, K. G. (1990). The wing beat of Drosophila melanogaster. II. Dynamics. Phil. Trans. R. Soc. Lond. B 327, $19-44$.

Zeil, J. (1983). Sexual dimorphism in the visual system of flies: The compound eyes and neural superposition in Bibionidae (Diptera). J. Comp. Physiol. A 150, 379-393. 